\section{Darwinism not tautological}

SIR - I would question both the ability and the need of Torres's work ${ }^{1}$ to defend the theory of natural selection from the accusation of tautology ${ }^{2}$. Torres presents a novel example of an evolutionary optimization model $^{3}$, in which the 'instantaneous fitness' of a set of phenotypic variants (in this case metabolic pathways) is defined according to a set of plausible rules (in this case thermodynamic criteria). All such models stand or fall on how well they predict the reality found in nature. If there is an anomaly, the model is modified $a$ posteriori, by changing the criteria, by introducing constraints to the phenotype set or by adding criteria from other models (for example, sexual selection criteria that result in traits being far from thermodynamically efficient in terms of individual survival). Thus, it can be argued, calling a phenotype 'instantaneously fit' equates to saying that it is there in the first place, and so we are back to the original tautology: 'survival of the survivors'.

The tautology argument is nevertheless flawed because, as many evolutionists since Darwin have reiterated, natural selection emerges as an inevitable consequence of observed facts and empirically derived precepts. To deny the existence of natural selection, therefore, is to deny that organisms depend on limited resources for their survival, that they reproduce with heritable variation, and that this variation will affect their ability to exploit those resources. It is worth noting that Popper no longer considers natural selection to be tautological ${ }^{4}$, although he still considers it to be a "metaphysical research program" in that to assume natural selection accounts for all evolutionary history (to the exclusion of other mechanisms) is more or less unfalsifiable. So far, however, no alternative fact-based mechanism is known that also accounts for the exquisite adaptation of organisms to their environment.

Department of Biology,

Michael. E. Weale

University of Southampton,

Bassett Crescent East,

Southampton SO9 3TU, UK

1. Torres, J.-L. /I Nuovo Cimento 13D, 177 (1991).

2. Maddox, J. Nature 350, 653 (1991).

3. Parker, G. A. \& Maynard Smith, J. Nature 348, 27-33 (1990)

4. Popper, K. R. Dialectica 32, 339-355 (1978),

SIR - John Maddox has raised once again the "nagging doubt" that the "theory of natural selection" is tautological. This is easily resolved once it is realized that natural selection is a mechanism, not a theory. In a system of replication with mutation, natural selection must occur. This is why Darwin called it "natural" selection, meaning "tautologically entailed" selection. The phrase "survival of the fittest" was introduced merely as an illustration for those who misunderstood the term natural selection, and is equally tauto- logical. Attempts to avoid the tautology by redefining "fitness" can succeed only by making the phrase false as well as falsifiable.

This, however, does not imperil the scientific status of Darwin's theory of evolution using natural selection. Darwin claimed, first, that evolution had occurred and, second, that natural selection was the mechanism responsible. Both of these propositions are testable and falsifiable and therefore scientific.

Coel Hellier

Mullard Space Science Laboratory, University College London,

Holmbury St Mary, Dorking,

Surrey RH5 6NT, UK

\section{Hinshelwood in the Pantheon?}

SIR - James D. Watson's obituary of Luria was a fitting tribute to a pioneer of molecular biology. There is no doubt that the Luria-Delbrück fluctuation test played a crucial role in establishing the spontaneous nature of mutations in bacteria, and made bacteria the organisms of choice for studying the nature of the gene.

However, contrary to Watson's remark, Cyril Hinshelwood did not argue against the mutational origin of resistant bacteria. Instead, he said there was another way they could become resistant, that is, through a change in their reaction pattern or chemical equilibrium. This he and his co-workers at Oxford established on the basis of the speed of change, the large fraction of the population involved, the finely graded nature of the response, the lack of precise specificity and the reversibility of the altered state ${ }^{2,3}$

Unfortunately, the work of the Oxford group coincided with the beginnings of the remarkable development of molecular genetics, and it seemed irrelevant at the time, particularly because the changes could not be identified with any macromolecule, in contrast to the elegant simplicity of genetic change.

Closer inspection shows that the adaptive changes described by the Oxford group were analogous to the Dauermodifikationen of Paramecium described by Jollos and confirmed by Sonneborn ${ }^{4}$. Similar findings have since been made in plant ${ }^{5}$ and animal cell culture $^{6-8}$, and appear to be central to the processes of differentiation and neoplastic transformation. Indeed, the adaptive changes of large cell populations may well be the most common heritable changes they undergo, if we accept an inheritance that persists for many generations upon removal of the inducing constraint, but is subject to gradual reversal.

Thus, while molecular genetics flourished as a result of Luria's work, adaptive change languished, resulting in a one-sided approach to the problems of development, ageing and cancer. I have no doubt that Hinshelwood will one day be placed on the same high level as Luria for his contribution to our understanding of the living state.

HARRY RUBIN

University of California, Berkeley,

Department of Molecular

and Cell Biology,

229 Stanley Hall,

Berkeley, California 94720, USA

1. Watson, J. D. Nature 350, 113 (1991).

2. Dean, A. C. R. \& Hinshelwood, C. Nature 199, 7-11 (1963)

3. Dean, A. C. R. \& Hinshelwood, C. Growth, Function and Regulation in Bacterial Cells (Oxford University Press. 1966).

Sonneborn, T. Adv. Genet. 1, 263-358 (1947)

5. Meins, F. Jr \& Binns, A. Proc. natn. Acad. Sci. U.S.A. 74 2928-2932 (1977).

6. Fox, M. \& Radicic, M. Mutat. Res. 49, 275-296 (1978). 7. Harris, M. Cell, 29, 483-492 (1982)

8. Rubin, A. L., Yao, A. \& Rubin, H. Proc. natn. Acad. Sci. U.S.A. $87,482-486(1990)$.

\section{Novel reading}

SIR - Steven Henikoff and Robert Levis (Nature 350, 9; 1991) suggest that the use of the word 'novel' in the biomedical literature is growing dramatically. Although this seems indeed to be the case, they do not look into possible explanations for this increase. As 'novel' means 'of a new kind; different from anything seen or known before', its use is certainly justifiable on occasion, and may often be preferable to words such as 'unique', which presupposes that no other examples will subsequently be found, or 'brand new', which suggests that it did not exist before its discovery. 'Somewhat atypical' might often be more accurate, but with journals setting limits on title and abstract length, 'novel' has an edge in being only five characters rather than 17.

The increased use of 'novel' may also be exaggerated in certain fields. From January 1989 to March 1991, only 1.1 per cent of all Medline citations use the word 'novel', but 3.5 per cent of citations on HIV (human immunodeficiency virus) claim novelty. Although HIV papers for the same period make up 2.3 per cent of the citations, 7.2 per cent of the 'novel' papers are on HIV. Given that there are now over 60,000 papers on HIV catalogued in Medline (nearly seven papers per nucleotide pair), it is difficult to believe that another few hundred novel discoveries will be made each year. This brings up another question: if an organism needs only $n$ nucleotide pairs completely to describe itself, then how many papers are required fully to understand the organism? Even the venerable bacteriophage T4 (which many people seem to think is 'solved') can claim only 1,226 citations ( 0.007 per nucleotide pair) in the entire Medline database since 1966

Alan C. Christensen Department of Biochemistry and

Molecular Biology,

Thomas Jefferson University,

Philadelphia, Pennsylvania 19107, USA 